\title{
Influência da gordura corporal no desempenho em saltos verticais em jogadores brasileiros de basquetebol
}

\author{
Influence of body fat on vertical jump performance of Brazilian professional basketball players
}

\author{
Vitor Luiz de Andrade ${ }^{1, *}$ \\ Ronaldo Bucken Gobbi ${ }^{1}$ \\ Danilo Rodrigues Bertucci ${ }^{1}$ \\ Pedro Balikian Junior ${ }^{2}$
}

\section{Resumo}

Objetivo: Investigar a composição corporal (CC) e o salto vertical bem como as possíveis correlações entre os parâmetros de CC e o desempenho de jogadores brasileiros de basquetebol. Métodos: Dez jogadores profissionais de basquetebol (idade $23,3 \pm 3,7$ anos, massa corporal 88,9 $\pm 15,3 \mathrm{~kg}$ e estatura $193 \pm 8,3 \mathrm{~cm}$ ) foram submetidos à análise da composição corporal (DXA) em que foram separados os segmentos corporais do tronco, pernas e total, e desempenho de salto vertical, mensurado pelo Squat Jump (SJ) e Countermovement Jump (CMJ). Resultados: Foram encontrados valores de percentual de gordura total (\%GTOTAL) de 16,4 $\pm 8,1 \%$ e massa gorda total (MGTOTAL) de 14,7 $\pm 9,3 \mathrm{~kg}$. Além disso, o percentual de gordura do tronco (\%GT) foi de $17,3 \pm 8,6 \%$ e massa gorda do tronco (MGT) foi de $7,4 \pm 4,9 \mathrm{~kg}$. O SJ $(37,7 \pm 5,7 \mathrm{~cm})$ foi significativamente menor que o CMJ $(40,8 \pm 6,0 \mathrm{~cm} ;+7,6 \pm$ $6,0 \% ; \mathrm{p}<0,05)$, que correspondeu a uma diferença absoluta (CMJ-SJ) de $3,1 \pm 2,7 \mathrm{~cm}$. Adicionalmente, foram encontradas correlações negativas entre o desempenho no CMJ e os parâmetros de \%GTOTAL e MGTOTAL, MGT e \%GT $(r=-0,69 ; r=-0,66 ; r=-0,71 ; r=-0,72 ; p<0,05)$. Conclusões: Jogadores de basquete brasileiros, a distribuição das massas (i.e., massa magra, massa gorda), principalmente a massa gorda total e a do tronco influencia diretamente no desempenho do salto.
\end{abstract}

Palavras-Chave: antropometria, DEXA, desempenho, basquetebol.

\begin{abstract}
Objective: Was to investigate the influence of body composition (BC) and vertical jump performance and possible correlations between BC parameters and performance of Brazilian professional basketball players. Methods: Ten professional basketball players $(23.3 \pm 3.7$ years, $88.9 \pm 15.3 \mathrm{~kg}$ and $193 \pm 8.3$ $\mathrm{cm}$ ) were submitted to body composition analysis (DXA) on the trunk, leg and total body segments, and vertical jump performance measured by Squat Jump (SJ) and Countermovement Jump (CMJ). Results: Total fat percentage (\% FTOTAL) and total fat mass (FMTOTAL) were $16.4 \pm 8.1 \%$ and $14.7 \pm 9.3 \mathrm{~kg}$, respectively. In addition, trunk fat percentage (\% TF) was $17.3 \pm 8.6 \%$ and trunk fat mass (TFM) was 7.4 $\pm 4.9 \mathrm{~kg}$. SJ $(37.7 \pm 5.7 \mathrm{~cm})$ was significantly lower than CMJ $(40.8 \pm 6.0 \mathrm{~cm},+7.6 \pm 6.0 \%, p<0.05)$, which corresponded to an absolute difference (CMJ-SJ) of $3.1 \pm 2.7 \mathrm{~cm}$. In addition, negative correlations were found between CMJ performance and \% FTOTAL and FMTOTAL, TFM and \% TF parameters $(r=-0.69, r=-0.66, r=-0.71, r=-0.72, p<0.05)$. Conclusion: Based on these results, it can be concluded that Brazilian professional basketball players, the mass distribution (i.e., lean mass, fat mass), especially total and torso fat mass have direct influences on jumping performance.
\end{abstract}

Keywords: anthropometry, DEXA, performance, basketball.
Afiliação dos autores

${ }^{1}$ Universidade Estadual Paulista, Rio Claro, São Paulo, Brasil.

${ }^{2}$ Universidade Federal de Alagoas, Maceió, Alagoas, Brasil.

${ }^{*}$ Autor correspondente

Programa de Pós Graduação em Ciências da Motricidade - Instituto de Biociências, Universidade Estadual Paulista, Av. 24 A,1515, Bela Vista, CEP 13506-900, Rio Claro, São Paulo, Brasil.

e-mail:

vitor.luiz.de.andrade@gmail.com

\section{Conflito de interesses}

Os autores declararam não haver conflito de interesses.

Processo de arbitragem

Recebido: 27/04/2017 Aprovado: 29/05/2017 


\section{Introdução}

O basquetebol é uma modalidade esportiva coletiva muito popular ao redor do mundo ${ }^{1}$. Entretanto, os Estados Unidos são considerados um país com muita tradição no esporte e disputa quase sempre os melhores lugares nas competições de alto nível mundial. No Brasil, embora o basquetebol ainda não tenha todo o apoio necessário, atualmente tem sido mais valorizado, principalmente pelas parcerias entre a Liga Nacional de Basquete (LNB) e a National Basketball Association (NBA). No Brasil, a produção científica no basquetebol ainda é pequena em relação aos grandes avanços alcançados em duas décadas de estudos feitos nos Estados Unidos ${ }^{1}$.

Neste sentido, apesar de existirem estudos no Brasil que mensuraram parâmetros antropométricos e de composição corporal (CC) em jogadores de basquetebol ${ }^{2}$, não há, para nosso conhecimento, estudos que objetivaram verificar a relação entre perfil de composição corporal e o desempenho em saltos verticais em jogadores adultos de alto nível do Brasil. Para adultos, o tempo de prática e experiência em diversos testes resultam em uma melhora nas habilidades motoras que são importantes para os atletas, particularmente para um jogador de basquetebol ${ }^{3}$. Além disso, a melhora das habilidades motoras também é acompanhada de uma melhora nos parâmetros relacionados à composição corporal bem como a energia dispendida para eventos de caráter de força ou potência ${ }^{4}$.

Atualmente, alguns estudos se preocuparam em investigar parâmetros antropométricos e somatotipo de praticantes de basquetebol ${ }^{5,6}$ juntamente com parâmetros fisiológicos relacionados à performance ${ }^{6,7,8}$ ao redor do mundo. Entretanto, fatores importantes relacionados à composição de segmentos corporais juntamente com as respostas e influencias sobre habilidades motoras essenciais como saltos verticais são pouco explorados na literatura específica para 0 basquetebol, principalmente em brasileiros.

Desta forma, o objetivo do estudo foi investigar os parâmetros de composição corporal nos segmentos envolvidos no salto vertical e as possíveis correlações entre os parâmetros de CC e o desempenho de jogadores brasileiros de basquetebol. A hipótese da presente investigação é que os parâmetros de CC e saltos verticais estejam próximos do que é encontrado na literatura específica para jogadores de basquetebol ao redor do mundo e que os parâmetros de CC tanto de maneira absoluta quanto de maneira segmentada pela massa muscular envolvida no salto vertical exerçam influência direta nos parâmetros de desempenho.

\section{Métodos}

\section{Participantes}

A amostra da presente investigação foi composta de dez jogadores profissionais de basquetebol (idade correspondente a $23,3 \pm 3,7$ anos, estatura $193 \pm 8,3 \mathrm{~cm}$ e massa corporal de $88,9 \pm 15,3 \mathrm{~kg}$ ) da categoria adulto pertencentes à Federação Paulista de Basquetebol (tabela 1). A posição dos jogadores compreendia em pivôs $(n=4)$, laterais $(n=4)$ e armadores $(n=$ 2). Os atletas possuíam no mínimo 5 anos de experiência em treinamentos e competições na modalidade. Treinavam entre 10 $-15 \mathrm{~h}$ sendo que tinham entre 5 e 7 sessões de treinamento em $5-6$ dias por semana. Os treinos eram compostos de exercícios de força, resistência aeróbia, resistência muscular localizada, treinos fundamentais, táticos e técnicos. Todos os procedimentos desta pesquisa foram aprovados pelo Comitê de Ética em Pesquisa da instituição e foram conduzidos concordando com a Declaração de Helsinki. Os atletas foram informados sobre os riscos e benefícios dos experimentos, e assinaram o termo de consentimento livre esclarecido antes do estudo.

\section{Delineamento Experimental}

As avaliações foram conduzidas durante dois dias separados por um intervalo de no máximo 48h. Durante os dias de avaliação, a temperatura e umidade relativa foram de aproximadamente $33^{\circ}$ e $60 \%$ respectivamente. No primeiro dia, os indivíduos visitaram o laboratório em horários marcados previamente e aleatoriamente. Neste dia, os participantes foram submetidos à estimativa da composição corporal por intermédio da técnica da absorptiometria de raios- $x$ de dupla energia (DEXA). No segundo dia, os indivíduos foram avaliados por meio da execução de três saltos verticais em duas modalidades distintas, Conter Moviment Jump (CMJ) e Squat Jump (SJ).

\section{Estimativa da composição corporal}

Para a análise da composição corporal (CC) foi utilizado um densitômetro (Lunar DPX-NT, GE Healthcare, Madison, WI, USA) bem como a distribuição das massas livre de gordura, massa gorda e massa óssea do corpo todo de acordo com a especificação do fabricante. A análise da distribuição das massas possibilitou a apresentação das massas magra e gorda total (MMTOTAL; MGTOTAL) bem como percentual de gordura total (\%GTOTAL). O delineamento em regiões de interesse foi realizado automaticamente por um software integrado (enCORE, GE Healthcare, Madison, WI, EUA; versão 11.40.004). Foram delineadas algumas regiões:

A perna, que foi definida a partir de um limite superior dado pela linha horizontal entre fêmur e a tíbia, um limite inferior dado pela linha horizontal através da articulação tíbio-talar e um limite lateral que foram considerados os cortes laterais exteriores da perna. Esta análise possibilitou a apresentação dos dados de massa magra e massa gorda, tanto da perna direita (MMMID; MGMID) quanto da perna esquerda (MMMIE; MGMIE), bem como o total (MMTMI; MGTMl;) e percentual de gordura total dos membros inferiores (\%GMI)

O tronco, que foi definido a partir de um limite superior dado pela linha horizontal sob a clavícula e o acrômio, um limite inferior dado pela linha horizontal sob a borda superior da espinha ilíaca ântero-superior e um limite lateral onde foram considerados os cortes laterais exteriores do tronco. Esta análise possibilitou a determinação da massa magra e gorda do tronco (MMT; MGT) bem como o percentual de gordura do tronco (\%GT).

Os resultados do densitômetro são expressos em gramas de massa magra, de gordura e percentual de gordura corporal bem como densidade mineral óssea $(\mathrm{g} \cdot \mathrm{cm} 2)$. Entretanto, todos os valores de CC serão expressos em $\mathrm{kg}$. Ao se posicionarem no aparelho, os avaliados permaneceram imóveis em posição de decúbito dorsal durante todo o exame (aproximadamente 15 $\min )$.

\section{Testes de salto verticais}

Para avaliação do salto vertical os atletas realizaram três saltos nas modalidades CMJ e SJ, separados por 30 segundos, e, entre as modalidades, um período de 2 minutos. Para tanto, utilizou-se uma plataforma de salto sensível a pequenas pressões (Axon Jump, bioengenharia desportiva, Buenos Aires, AR) ligada a um computador com software específico (Axon Jump 4.0, bioengenharia desportiva, Buenos Aires, AR), sendo utilizada a maior altura atingida entre os saltos.

Para o SJ os atletas foram orientados a posicionarem-se em preparação ao salto, com as articulações dos quadris e joelhos flexionadas a $90^{\circ}$, sendo mantida esta posição por $10 \mathrm{~s}$ e ao sinal, executarem o salto vertical a partir desta posição inicial (apenas movimento ascendente) em máximo esforço enquanto no CMJ partiram da posição estendida e realizaram rápido movimento de preparação descendente, flexionando as articulações dos quadris e joelhos previamente ao movimento ascendente em máximo esforço. Em todas as modalidades de salto as mãos foram posicionadas na cintura e não foi permitido - uso delas durante as avaliações. Através da diferença absoluta das alturas do CMJ e SJ pode-se determinar um índice de aproveitamento da energia elástica (CMJ-SJ) segundo o estudo de Smirniotou et al. ${ }^{9}$.

\section{Análise estatística}

A normalidade e homogeneidade dos dados foram testadas por meio do teste de Kolmogorov-Smirnov \& Liliefors test. A confirmação da normalidade possibilitou a apresentação dos dados por meio da média e desvio padrão. As possíveis diferenças entre as massas magra e gorda dos membros direito (MMMID; MGMID) e esquerdo (MMMIE; MGMIE) bem como a altura dos saltos SJ e CMJ foram testadas a partir do teste t de Student para amostras dependentes. As possíveis correlações entre as variáveis de desempenho no salto vertical (SJ; CMJ; 
CMJ-SJ) com as variáveis de CC foram testadas a partir do teste de correlação de Pearson. O valor de $p$ foi fixado em todos os casos para menor que $5 \%$.

\section{Resultados}

A análise da normalidade permitiu a apresentação da estatística descritiva por meio de média e desvio padrão, bem como o intervalo de confiança de 95\% (IC 95\%) das medidas corporais tanto de maneira absoluta (i.e., total) quanto por segmentos envolvidos (i.e., membros inferiores e tronco). Também serão apresentados os valores médios e desvio padrão da altura de salto obtida durante os dois testes de salto vertical (i.e., SJ e CMJ). Na tabela 1 estão apresentados os valores de CC total dos sujeitos do estudo.

Tabela 1

Características físicas dos sujeitos e os valores de CC adquiridos por meio do DEXA $(n=10)$.

\begin{tabular}{lrcc}
\hline Variável & Média & Desvio padrão & \multicolumn{1}{c}{ IC 95\% } \\
\hline Idade (anos) & 23,3 & 3,7 & $20,7-25,9$ \\
Estatura $(\mathrm{cm})$ & 192,9 & 8,3 & $187,0-198,9$ \\
Massa total $(\mathrm{kg})$ & 88,5 & 14,9 & $77,9-99,2$ \\
\%GTOTAL $(\%)$ & 16,4 & 8,1 & $10,6-22,2$ \\
MMTOTAL $(\mathrm{kg})$ & 69,5 & 6,7 & $64,5-74,4$ \\
MGTOTAL $(\mathrm{kg})$ & 14,7 & 9,3 & $8,0-21,3$ \\
\hline Percentual de gordura total & $=$ \%GTOTAL; & Massa magra total $=$ MMTOTAL;
\end{tabular}

Percentual de gordura total
Massa gorda total MGTOTAL.

Na Tabela 2 estão os valores de CC dos membros inferiores do lado direito e esquerdo, total dos membros inferiores e tronco.

Tabela 2

Características da CC dos membros inferiores e do tronco dos sujeitos ( $\mathrm{n}$ $=10)$.

\begin{tabular}{lccc}
\hline Variável & Média & Desvio padrão & IC 95\% \\
\hline MMMID & 12,7 & 1,7 & $11,5-13,9$ \\
MGMID & 3,1 & 1,9 & $1,7-4,5$ \\
MMMIE & 12,7 & 1,6 & $11,6-13,8$ \\
MGMIE & 3,1 & 1,9 & $1,7-4,5$ \\
MMTMI & 25,4 & 3,2 & $23,0-27,7$ \\
MGTMI & 6,2 & 4,0 & $3,3-9,0$ \\
\%GMI & 18,0 & 8,4 & $12,0-24,1$ \\
MMT & 30,7 & 2,9 & $28,7-32,8$ \\
MGT & 7,4 & 4,9 & $3,9-10,8$ \\
\%GT & 17,3 & 8,6 & $11,1-23,4$ \\
\hline
\end{tabular}

Massa magra do membro inferior direito = MMMID; Massa gorda do membro inferior direito = MGMID; Massa magra do membro inferior esquerdo = MMMIE; Massa gorda do membro inferior esquerdo = MGMIE; Massa magra total dos membros inferiores = MMTMI; Massa gorda total dos membros inferiores = membros inferiores = MMTMl; Massa gorda total dos membros inferiores =
MGTMI; Percentual de gordura dos membros inferiores = \%GMI; Massa magra do tronco = MMT; Massa gorda do tronco = MGT; Percentual de gordura do tronco $=\% \mathrm{GT}$.

Não foram encontradas diferenças significativas entre MMMI tanto do lado direito quanto esquerdo, bem como, também não foram encontradas diferenças entre MGMI entre os lados $(p>0,05)$. Além disso, a MMMID apresentou forte correlação com MMMIE $(r=0,95 ; p<0,001)$ e a MGMID também apresentou forte correlação com a MGMIE $(r=0,99 ; p<0,001)$. O SJ $(37,7 \pm 5,7$ $\mathrm{cm})$ foi significativamente menor que o CMJ $(40,8 \pm 6,0 \mathrm{~cm} ;+7,6$ $\pm 6,0 \% ; p<0,05)$, que correspondeu a uma diferença absoluta (CMJ-SJ) de 3,1 $\pm 2,7 \mathrm{~cm}$ (Figura 1).

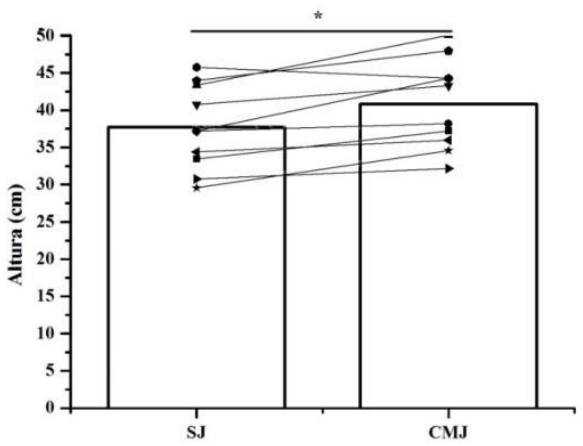

Figura 1. Em barra o comportamento médio da altura dos saltos verticais (i.e.,SJ e CMJ). Em símbolo+linha, o comportamento individual dos saltos verticais dos participantes. ${ }^{*}$ Significa diferença signficativa na altura dos saltos $(p<0,05)$.
Foram encontradas correlações significativas entre 0 desempenho nos saltos verticais (i.e., SJ e CMJ) com a \%GTOTAL, MGTOTAL, MGT e \%GT (Figura 2). Não foram encontradas correlações entre a CC e o parâmetro de CMJ-SJ.
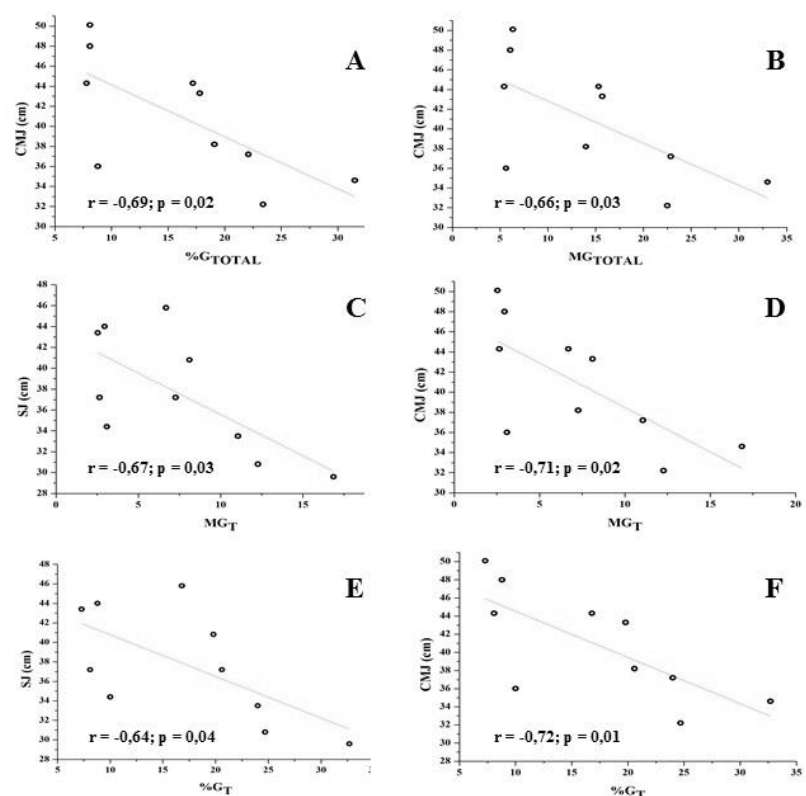

Figura 2. Correlações obtidas entre o desempenho nos saltos verticais SJ e CMJ) com a CC. A = Correlação entre o percentual de gordura total (\%GTOTAL) e a altura do $\mathrm{CMJ}$; $\mathrm{B}=$ Correlação entre a massa gorda total (MGTOTAL; kg) e a altura do CMJ; C = Correlação entre a massa gorda do tronco (MGT; kg) e a altura do SJ; D = Correlação entre a massa gorda do tronco (MGT; kg) e a altura do $\mathrm{CMJ} ; \mathrm{E}$ = Correlação entre o percentual de gordura do tronco (\%GT; $\mathrm{kg}$ ) e a altura do SJ; F = Correlação entre o percentual de gordura do tronco (\%GT; kg) e a altura do CMJ.

\section{Discussão}

Os principais achados da presente investigação foram a não existência de diferença entre a CC dos membros inferiores de jogadores brasileiros profissionais de basquetebol. Além disso, houve uma diferença significativa entre as modalidades do salto vertical (i.e., SJ e CMJ). Entretanto, pode-se considerar que o comportamento individual dos participantes revelou em pelo menos um caso que o salto vertical SJ foi maior que o CMJ. Adicionalmente, um outro achado importante da presente investigação foram as correlações entre a \%GTOTAL, MGTOTAL, MGT e \%GT com o desempenho no SJ e no CMJ.

A hipótese do estudo era que os valores de $\mathrm{CC}$ de jogadores brasileiros de basquetebol estariam próximos do que é encontrado na literatura específica e que os parâmetros de composição dos membros inferiores influenciassem diretamente o desempenho do salto. Esta hipótese foi refutada pelos resultados evidenciados na literatura, principalmente pelas características de CC total que no presente estudo responderam negativamente ao que é mostrado na literatura internacional. Além disso, os parâmetros de CC dos membros inferiores não apresentaram correlações significativas com os saltos verticais. Entretanto, foram encontradas significativas correlações entre a MGT e \%GT com o desempenho nos saltos.

Em jogadores de alto nível que disputavam a NBA e compunham a seleção olímpica dos Estados Unidos foram encontrados valores de \%GTOTAL de $11,5 \pm 4,6 \%{ }^{7}$. Em jogadores da Servia foram encontrados valores de \%GTOTAL de $11,5 \pm 1,9 \%{ }^{10}$. Na Bélgica, foram encontrados valores de $\%$ GTOTAL que variam entre as posições de $11,2 \pm 2,8 \%$ até $15,2 \pm 4,0 \%{ }^{11}$. Na Tunísia, foram encontrados valores de $\%$ GTOTAL em 2007 de $6,1 \pm 3,7 \%$ até $10,4 \pm 7,8 \%$ dependendo da posição do jogador ${ }^{12}$, em 2010 os valores de \%GTOTAL foram de $8,3 \pm 1,6 \%$ até $14,8 \pm 1,9$ também dependendo da posição do jogador ${ }^{13}$. Na França, foram encontrados valores de $\%$ GTOTAL de $13,5 \pm 0,35 \%$ até $13,7 \pm 0,51 \%^{14}$. Em Portugal, jogadores da categoria juniores obtiveram um perfil semelhante aos jogadores adultos dos Estados Unidos e Servia, com valores 
de $\% G_{\text {TOTAL }}$ de $11,5 \pm 3,3 \%{ }^{15}$, na elite júnior portuguesa, também foram encontrados valores de $\% \mathrm{G}_{\text {TOTAL }}$ de $12,7 \pm 1,7 \%{ }^{4}$.

$\mathrm{Na}$ presente investigação foram encontrados valores de $\% \mathrm{G}_{\text {TOTAL }}$ de $16,4 \pm 8,1 \%$ que podem ser considerados elevados considerando os valores que já foram encontrados ao em outras regiões. Entretanto, em jogadores brasileiros recentemente foi encontrado valores de $\% \mathrm{G}_{\text {TOTA }} \mathrm{L}$ de $26,1 \pm 8,3 \%{ }^{16}$. Embora estes resultados tenham sido mensurados de uma maneira menos sensível (e.g., método por dobras cutâneas) ${ }^{17}$, pode-se destacar que em brasileiros, os resultados mostram um perfil de \% $\mathrm{G}_{\text {TOTAL }}$ em desvantagem, considerando resultados encontrados na literatura. Além disso, quando analisados os valores de $\mathrm{MG}_{\text {TOTAL, }}$, foram encontrados em jogadores portugueses uma $\mathrm{MG}_{\text {TOTAL }}$ de $9,0 \pm 2,8 \mathrm{~kg}^{15}$ e $9,8 \pm 2,1 \mathrm{~kg}^{4}$. Isso destaca a desvantagem morfológica dos jogadores brasileiros que precisariam de mais potência muscular e consequentemente maior demanda energética para realizar uma atividade, haja vista a maior MG TOTAL. A massa gorda é considerada um peso adiacional e sem funcionalidade em atividades esportivas (i.e., salto vertical, locomoção) principalmente em esportes onde ocorre o deslocamento $^{18}$

Além disso, quando analisados os parâmetros de $\mathrm{MM}_{\mathrm{TOTAL}}$, foram encontrados valores que variam de $67,0 \pm 5,2 \mathrm{~kg}^{4}$ até 69,4 $\pm 6,3 \mathrm{~kg}^{15} \mathrm{em}$ Portugueses, enquanto que em Europeus, foram encontrados valores de MM TOTAL de 77,2 $\pm 8,5 \mathrm{~kg}^{5}$. Adicionalmente aos resultados apresentados, cabe ressaltar que nos estudos supracitados, os parâmetros antropométricos de estatura, massa corporal e idade são semelhantes aos apresentados aqui e que estes resultados suportam ainda mais a ideia de que os jogadores brasileiros estão em desvantagem em relação a CC encontrada pelo mundo, haja vista os resultados apresentados.

Apenas um estudo de nosso conhecimento mostrou alguns parâmetros de composição corporal dos membros inferiores e tronco em jogadores de basquetebol ${ }^{4}$. A MMTMI da presente investigação $(25,4 \pm 3,2 \mathrm{~kg})$ apresentou ser superior ao encontrado por Silva et al. ${ }^{4}$ que mostraram $22,7 \pm 2,0 \mathrm{~kg}$, entretanto, a MGTMI apresentou-se menor $(4,0 \pm 1,1 \mathrm{~kg})^{4} \mathrm{ao}$ encontrado na presente investigação $(6,2 \pm 4,0 \mathrm{~kg})$. Além disso, $0 \% \mathrm{GMI}$ apresentou-se superior na presente investigação (18,0 $\pm 8,4 \%$ ), enquanto Silva et al. ${ }^{4}$ mostraram $14,3 \pm 2,7 \%$.

Quando analisado o tronco, Silva et al. ${ }^{4}$ mostraram que a composição do tronco era composta de $30,4 \pm 2,7 \mathrm{~kg}$ de MMT, $3,8 \pm 0,9 \mathrm{~kg}$ de MGT que correspondeu a $10,7 \pm 1,6 \%$ de $\%$ GT. $\mathrm{Na}$ presente investigação foi encontrado valor semelhante de MMT $(30,7 \pm 2,9 \mathrm{~kg})$, no entanto, a MGT foi muito superior $(7,4 \pm$ $4,9 \mathrm{~kg}$ ) ao encontrado por Silva et al. ${ }^{4}$, além disso, o \%GT também foi muito superior $(17,3 \pm 8,6 \%)$. Estes resultados reforçam a existência de desvantagem dos jogadores brasileiros, sendo as regiões mais determinantes para execução da locomoção e saltos verticais que apresentaram acumulo de gordura.

O desempenho em saltos verticais são amplamente divulgados, principalmente pela facilidade de aplicação dos testes bem como, pela aplicabilidade e sensibilidade aos efeitos do treinamento ${ }^{19}$. Soares et $a .^{20} \mathrm{em}$ um estudo antigo, encontraram em brasileiros valores de salto vertical de 55,9 \pm $8,1 \mathrm{~cm}$ até $66,8 \pm 8,3 \mathrm{~cm}$ dependendo da posição do jogador. Para jogadores da NBA e seleção olímpica dos Estados Unidos, foram encontrados valores de salto vertical de $57,4 \pm 7,7 \mathrm{~cm}^{7}$. $\mathrm{Na}$ NationalCollegiate Athletic Association (NCAA) foram encontrados valores de salto vertical de $66,0 \pm 6,9 \mathrm{~cm}$ até $72,6 \pm$ $5,6 \mathrm{~cm}$ entre os anos de 1988 a $1992^{21}$. Entretanto, nos estudos supracitados, foi permitido o uso dos braços o que não foi permitido na presente investigação.

Quando analisados estudos anteriores que utilizaram de uma metodologia semelhante, na Bélgica, foram encontrados valores de $35,7 \pm 3,2 \mathrm{~cm}$ até $41,0 \pm 3,8 \mathrm{~cm}$ para o SJ e $36,2 \pm$ $4,1 \mathrm{~cm}$ até $42,7 \pm 3,8 \mathrm{~cm}$ para o $\mathrm{CMJ}^{11}$. Em portugueses foram encontrados $35,6 \pm 4,4 \mathrm{~cm}$ para $\mathrm{CMJ}^{15}$. Em brasileiros, os estudos realizados permitiam a utilização dos braços durante a avaliação ${ }^{16,20}$ o que dificulta a comparação entre os resultados. Entretanto, mesmo assim, recentemente Borganha et al. ${ }^{16}$ apresentaram valores de $39,8 \pm 7,2 \mathrm{~cm}$ no salto vertical. Estes resultados demonstram que mesmo com diferenças metodológicas, ainda é possível dizer que nossos resultados para avaliação do desempenho do salto estão condizentes com a literatura específica.
Desse modo, sabendo que a CC pode ser um fator indispensável para um melhor desempenho e que em esportes coletivos (i.e., basquetebol e voleibol) a força explosiva é fundamental ${ }^{18,22}$. A combinação entre estes fatores teriam uma importância ótima para 0 melhor desempenho. Foram encontradas correlações entre a massa gorda e percentual de gordura total e no tronco (i.e., \% $\mathrm{G}_{\text {TOTAL }}, \mathrm{MG}_{\mathrm{TOTAL}}$, MGT e \%GT) com o desempenho nos saltos verticais. Estes achados se repetem na literatura em relação a movimentos explosivos ${ }^{23}$ entretanto, de nosso conhecimento para o basquetebol, este tipo de análise ainda não foi tão bem explorada.

As correlações negativas encontradas mostram o peso morto carregado pelo indivíduo tanto no total, quanto no tronco, podem contribuir negativamente para o desempenho. Sabendo que a gordura acumulada no homem está mais situada no abdômen e visceral ${ }^{24}$. Torna-se muito importante para o desempenho, em relação aos nossos resultados, um cuidado maior com a gordura acumulada no tronco e no total, bem como o percentual total encontrado. Nós observamos na literatura que os jogadores brasileiros apresentam uma distribuição da composicão corporal (i.e., massa magra, massa gorda) menos favorecida. Dessa maneira, o desempenho do brasileiro parece sofrer influência dos parâmetros de CC diretamente, o que pode determinar os padrões de deslocamento tanto verticais quanto horizontais durante uma partida. Principalmente pelas descrições na literatura em relação às correlacões com desempenho em corridas curtas e saltos verticais ${ }^{23,25-27}$

\section{Conclusão}

Com base nos nossos resultados, pode-se concluir que os parâmetros de composição corporal envolvidos no salto vertical não apresentaram diferença significativa, bem como não foram encontradas correlações com o desempenho. Entretanto, com base na literatura específica, os parâmetros de composição corporal de basquetebolistas brasileiros estão abaixo do que foi relatado em pesquisas ao redor do mundo e o desempenho dos saltos verticais está de acordo com a literatura anterior. Além disso, nossos achados suportam que em jogadores de basquete brasileiros, a distribuição das massas (i.e., massa magra, massa gorda) influenciam diretamente no desempenho do salto, principalmente massa gorda total e do tronco.

\section{Referências}

1. Ostojic SM, Mazic S, and Dikic N. Profiling in basketball: physical and physiological characteristics of elite players. J Strength Cond Res 20: 740744, 2006.

2. Paiva Neto A and César MC. Body composition assessment in male basketball players in brazilian national basketball league 2003. BrazilianJournalofKinanthropometryandHuman Performance 7: 35 - 44 2005

3. Litkowycz R, Olex-Zarychta D, Grygorowicz M, and Dzienisiewicz I. Withdrawal from sport training of young talented basketball players and its impact on selection in sport. Trends in Sport Sciences 2: 111-120, 2014.

4. Silva AM, Santos DA, Matias CN, Rocha PM, Petroski EL, Minderico CS, and Sardinha LB. Changes in regional body composition explain increases in energy expenditure in elite junior basketball players over the season. Eur J ApplPhysiol 112: 2727-2737, 2012

5. Nikolaidis P, Calleja-González J, and Padulo J. The effect of age on positional differences in anthropometry, body composition, physique and anaerobic power of elite basketball players. Sport Science and Health, 2014.

6. Boone $\mathrm{J}$ and Bourgois $\mathrm{J}$. Morphological and physiological profile of elite basketball players in Belgium. Internationaljournalofsportsphysiologyand performance 8: 630 - 638, 2013

7. Ostojic SM, Mazic S, and Dikic N. Profiling in basketball: physical and physiological characteristics of elite players. J StrengthCond Res 20: 740 744, 2006

8. Sallet P, Perrier D, Ferret JM, Vitelli V and Baverel G. Physiological differences in professional basketball players as a function of playing position and level of play. The Journalofsports medicine andphysical fitness 45: $291-294,2005$

9. Smirniotou A, Katsikas C, Paradisis G, Argeitaki P, Zacharogiannis E, and Tziortzis S. Strength-power parameters as predictors of sprinting performance. The Journalofsports medicine andphysical fitness 48: 447454, 2008.

10. Popovic S, Bjelica D, Jaksic D, and Hadzic R. Comparative Study of Anthropometric Measurement and Body Composition between Elite Socce and Volleyball Players. InternationalJournalofMorphology 32: 267 - 274 2014

11. Boone $\mathrm{J}$ and Bourgois $\mathrm{J}$. Morphological and physiological profile of elite basketball players in Belgian. Internationaljournalofsportsphysiologyand performance 8: 630-638, 2013.

12. Ben Abdelkrim N, El Fazaa S, and El Ati J. Time-motion analysis and physiological data of elite under-19-year-old basketball players during 
competition. British journalofsports medicine 41: 69-75; discussion 75, 2007.

3. Ben Abdelkrim N, Castagna C, Jabri I, Battikh T, El Fazaa S, and Ati JE. Activity Profile and Physiological Requirements of Junior Elite Basketball Players in Relation to Aerobic-Anaerobic Fitness. The JournalofStrength\&ConditioningResearch 24: 2330-2342, 2010.

14. Cormery $B$, Marcil $M$, and Bouvard $M$. Rule change incidence on physiological characteristics of elite basketball players: a 10-year-period investigation. British journalofsports medicine 42: 25-30, 2008.

15. Santos DA, Matias CN, Rocha PM, Minderico CS, Allison DB, Sardinha LB, and Silva AM. Association of basketball season with body composition in elite junior players. The Journalofsports medicine andphysical fitness 54: 162-173, 2014.

16. Bonganha V, Cavaglieri CR, Daniel JF, Mercadante LA, Montagner PC, and Borin JP. Neuromuscular and metabolic characteristics of elite basketball referees. The Journalofsports medicine andphysical fitness 53 : 217-223, 2013

17. Kulkarni B, Mamidi RS, Balakrishna N, and Radhakrishna KV. Body composition assessment in infancy and early childhood: comparison of anthropometry with dual-energy X-ray absorptiometry in low-income group children from India. Europeanjournalofclinicalnutrition 68: 658-663, 2014.

18. Mala L, Maly T, Zahalka F, Bunc V, Kaplan A, Jebavy R, and Tuma M. Body composition of elite female players in five different sports games. Journalofhumankinetics 45: 207-215, 2015.

19. Ramachandran S and Pradhan B. Effects of short-term two weeks low intensity plyometrics combined with dynamic stretching training in improving vertical jump height and agility on trained basketball players. Indianjournalofphysiologyandpharmacology 58: 133-136, 2014.

20. Soares J, Mendes OC, Neto CB, andMatisudo VKR. Physical fitness characteristics of Brazilian national basketball team as related to game functions. In: Perspectives in Kinathropometry JAP Day, ed Champaign, IL: Human Kinetics, : 127 - 133, 1986.

21. Hoffman JR, Tenenbaum G, Maresh CM, and Kraemer WJ. Relationship Between Athletic Performance Tests and Playing Time in Elite College Basketball Players. The JournalofStrength\&ConditioningResearch 10: 6771, 1996.

22. Alemdaroglu U. The relationship between muscle strength, anaerobic performance, agility, sprint ability and vertical jump performance in professional basketball players. Journalofhumankinetics 31: 149-158, 2012.

23. Sporis G, Vuleta D, Vuleta D, Jr., and Milanovic D. Fitness profiling in handball: physical and physiological characteristics of elite players. Collegiumantropologicum 34: 1009-1014, 2010.

24. Sala ML, Roell B, van der Bijl N, van der Grond J, de Craen AJ, Slagboom EP, van der Geest R, de Roos A, andKroft LJ. Genetically determined prospect to become long-lived is associated with less abdominal fat and in particular less abdominal visceral fat in men. Age andageing, 2015.

25. Dardouri W, Selmi MA, Sassi RH, Gharbi Z, Rebhi A, Yahmed MH, and Moalla W. Relationship Between Repeated Sprint Performance and both Aerobic and Anaerobic Fitness. Journalofhumankinetics 40: 139-148, 2014

26. Loturco I, Pereira LA, Cal Abad CC, D'Angelo RA, Fernandes V, Kitamura $\mathrm{K}$, Kobal R, and Nakamura FY. VERTICAL AND HORIZONTAL JUMP TESTS ARE STRONGLY ASSOCIATED WITH COMPETITIVE PERFORMANCE IN 100-M DASH EVENTS. J StrengthCond Res, 2015.

27. Moncef $C$, Said M, Olfa N, and Dagbaji G. Influence of morphological characteristics on physical and physiological performances of tunisian elite male handball players. Asianjournalofsports medicine 3: 74-80, 2012. 